\title{
Persistence and smoothness of critical relative elements in Hamiltonian systems with symmetry
}

\author{
Juan-Pablo ORTEGA and Tudor S. RATIU \\ Department of Mathematics, University of California, Santa Cruz, \\ Santa Cruz, CA 95064, USA; \\ E-mail: jportega@rats.uesc.edu, ratiu@cats.uese.edu
}

\begin{abstract}
We use new global tools in singular reduction in order to generalize some results on the persistence and smoothness of critical relative elements in Hamiltonian systems with symmetry, obtained by Montaldi (see [5] and [4]) and Patrick (see [8]), to the case in which the elements considered have non-trivial symmetry.

Persistance et différentiabilité

de l'ensemble des éléments critiques relatifs

dans les systèmes hamiltoniens symétriques

Résumé. De nouveaux outils globaux dans la théorie de réduction singulière sont utilisés pour généraliser les résultats de Montaldi (voir [5] et [4]) et Patrick (voir [8]) sur la persistance et la différentiabilité des éléments critiques relatifs dans les systèmes hamiltoniens symétriques si ces éléments possèdent une symétrie non triviale.
\end{abstract}

\section{Version française abrégée}

Soit $\left(M, \omega, \mathbf{J}: M \rightarrow \mathfrak{g}^{*}\right)$ un système hamiltonien avec la symétrie donnée par un groupe de Lie $G$ qui agit d'une manière propre sur $M$, où $\mathfrak{g}$ est l'algèbre de Lie de $G, \mathfrak{g}^{*}$ est le dual de $\mathfrak{g}$ et $\mathbf{J}$ est l'application moment équivariante. Si $h: M \rightarrow \mathbb{R}$ est un hamiltonien $G$-invariant, un équilibre relatif de $h$ est un point $m \in M$ tel que la courbe intégrale $m(t)$ du champ vectoriel hamiltonien $X_{h}$, $m(0)=m$, coïncide avec $\exp (t \xi)$ pour quelque $\xi \in \mathfrak{g}$. Un théorème de Montaldi (voir [5]) affirme que si l'action est libre, $G$ est compact et $m \in \mathbf{J}^{-1}(\mu)$ est un équilibre relatif non-dégénéré de $h$, il persiste lorsqu'on change $\mu$ et, en plus, on peut donner une limite inférieure pour le nombre de ces équilibres relatifs perturbés en termes du groupe de Weyl de $G$. La situation est analogue pour les orbites périodiques relatives (voir [4]) - orbites $\gamma(t)$ telles qu'il existe $g \in G$ et $T>0$ (la période) avec $\gamma(t+T)=g \cdot \gamma(t)$. Un autre résultat intéressant sur la structure de l'ensemble des équilibres

Note présentée par Charles-Michel MARLE. 


\section{J.-P. Ortega and T. S. Ratiu}

relatifs a été donné par Patrick dans [8] : si l'action de $G$ est libre et $G$ est compact, l'ensemble des équilibres relatifs autour d'un équilibre donné, avec moment et vitesse réguliers, est une variété symplectique de dimension $\operatorname{dim} G+\operatorname{rang} G$.

Nous allons généraliser ces résultats au cas où les éléments critiques considérés ont une symétrie non triviale, c'est-à-dire le groupe d'isotropie $H:=G_{m}$ de $m$ est un sous-groupe non trivial de $G$ avec normalisateur $\mathrm{N}(H)$ compact. Le premier outil dont nous avons besoin est l'analogue de l'espace réduit de Marsden et Weinstein (voir [3]), $M_{\mu}:=\mathbf{J}^{-1}(\mu) / G_{\mu}$, dans le cas singulier. On peut démontrer (voir [10], [2] et [7]) que l'espace topologique $M_{\mu}$ est stratifié par les variétés symplectiques $M_{\mu}^{(H)}:=\mathbf{J}^{-1}(\mu) \cap M_{(H)}^{G_{\mu} / G_{\mu}}$ associées aux types orbitaux $(H)$ de $G$. La structure symplectique et la dynamique réduite sur $M_{\mu}^{(H)}$ sont définies d'une manière analogue au cas régulier. Le symbole $M_{(H)}^{G_{\mu}}$ désigne l'ensemble des éléments dans $M$ dont le groupe d'isotropie est un sous-groupe conjugué à $H$ dans $G_{\mu}$.

Cet espace réduit $M_{\mu}^{(H)}$ est symplectomorphe à l'espace obtenu par réduction de Marsden-Weinstein, associé à l'action du groupe $L=\mathrm{N}(H) / H$ sur la sous-variété symplectique $M_{H}$ - l'ensemble des éléments dans $M$ dont le groupe d'isotropie est $H$. Il s'avère que les estimations de Montaldi sur la persistance se généralisent au cas singulier si on remplace le groupe de Weyl de $G_{\mu}$ par le groupe de Weyl de $\mathrm{N}_{G_{H}}(H) / H$. La situation est similaire avec le théorème de Patrick, où on remplace $G$ par $\mathrm{N}(H) / H$.

Les ingrédients principaux que nous utiliserons sont des modèles globaux pour les espaces réduits, décrits en détail dans [7]. Ces modèles nous permettent de réduire notre recherche sur les éléments critiques relatifs singuliers au cas régulier.

\section{Introduction and results on singular reduction}

Let $\left(M, \omega, G, \mathbf{J}: M \rightarrow \mathfrak{g}^{*}, h: M \rightarrow \mathbb{R}\right)$ be a Hamiltonian dynamical system with a symmetry given by the Lie group $G$ acting properly over $M$ and corresponding equivariant momentum map $\mathbf{J}$, where $\mathfrak{g}$ is the Lie algebra of $G$ and $\mathfrak{g}^{*}$ is its dual. Recall that a relative equilibrium of $h$ is a point $m \in M$ such that the integral curve $m(t)$ of the Hamiltonian vector field $X_{h}$ starting at $m$ equals $\exp (t \xi)$ for some $\xi \in \mathfrak{g}$, where $\exp : \mathfrak{g} \rightarrow G$ is the exponential map and $\xi$ is the velocity of the relative equilibrium. It has been shown by Montaldi in [5] that if the group action is free, $G$ is compact, and $m \in \mathbf{J}^{-1}(\mu)$ is a non-degenerate relative equilibrium of $h$, this relative equilibrium persists to nearby level sets of the momentum map; he also gives a lower bound for the number of these bifurcated relative equilibria in terms of the Weyl group of $G$. This problem, with different hypotheses, has also been studied in [9] and [6]. The same can be done with relative periodic orbits (RPOs), that is, trajectories $\gamma(t)$ for which $\gamma(t+T)=g \cdot \gamma(t)$ for some $g \in G$ and some $T>0$ (the period). Another remarkable result dealing with the structure of the set of relative equilibria of a Hamiltonian system is due to Patrick in [8], who proved that if $G$ is compact and acts freely, the set of relative equilibria around a given one with regular momentum and velocity, is locally a symplectic manifold of dimension $\operatorname{dim} G+\operatorname{rank} G$.

We will generalize these results to the case in which the critical elements under consideration have non-trivial symmetry, that is, the stabilizer $H:=G_{m}$ is a non-trivial subgroup of $G$, with compact normalizer $\mathbf{N}(H)$. In this case a good understanding of singular reduction is critical. We briefly enumerate below the results needed, in the formulation of [7], most appropriate for our purposes. For the statements that follow and additional information, see [10], [2], and [7]. 
(i) If $H:=G_{m} \neq\{e\}$, we will replace the Marsden-Weinstein reduced space (see [3]) $M_{\mu}=\mathrm{J}^{-1}(\mu) / G_{\mu}$ by the set $M_{\mu}^{(H)}:=\mathbf{J}^{-1}(\mu) \cap M_{(H)}^{G_{\mu} / G_{\mu}} ; \mathfrak{h}$ will denote the Lie algebra of $H$. We let $M_{(H)}^{G_{\mu}}:=\left\{z \in M \mid G_{z}=g H g^{-1}\right.$ for some $\left.g \in G_{\mu}\right\}$ be the set of points in $M$ whose isotropy is conjugate in $G_{\mu}$ to the subgroup $H$ of $G$. The set $M_{\mu}^{(H)}$ has a unique differentiable structure such that the canonical projection

$$
\pi_{\mu}^{(H)}: \mathbf{J}^{-1}(\mu) \cap M_{(H)}^{G_{\mu}} \longrightarrow M_{\mu}^{(H)}
$$

is a surjective submersion. The differentiable manifold $M_{\mu}^{(H)}$ is diffeomorphic to $\left(\mathbf{J}^{-1}(\mu) \cap\right.$ $\left.M_{H}\right) /\left(\mathrm{N}_{G_{\mu}}(H) /(H)\right)$, where $M_{H}:=\left\{z \in M \mid G_{z}=H\right\}$ and $\mathrm{N}_{G_{\mu}}(H)=\left\{n \in G_{\mu} \mid n H n^{-1}=H\right\}$. As expected, there is a unique symplectic structure $\omega_{\mu}^{(H)}$ on $M_{\mu}^{(H)}$ characterized by:

$$
i_{\mu}^{(H) *} \omega=\pi_{\mu}^{(H) *} \omega_{\mu}^{(H)},
$$

where $i_{\mu}^{(H)}: \mathbf{J}^{-1}(\mu) \cap M_{(H)}^{G_{\mu}} \hookrightarrow M$ is the inclusion.

(ii) The dynamics induced by the $G$-invariant Hamiltonian $h$ reduces consistently with this singular reduction scheme, namely the flow $F_{t}$ of $X_{h}$ leaves the connected components of $\mathbf{J}^{-1}(\mu) \cap M_{(H)}^{G_{\mu}}$ invariant and commutes with the $G_{\mu}$-action, so it induces a flow $F_{t}^{\mu}$ on $M_{\mu}^{(H)}$ that satisfies $\pi_{\mu}^{(H)} \circ F_{t}=F_{t}^{\mu} \circ \pi_{\mu}^{(H)}$. This flow $F_{t}^{\mu}$ is Hamiltonian on $M_{\mu}^{(H)}$, with Hamiltonian function $h_{\mu}^{(H)}: M_{\mu}^{(H)} \rightarrow \mathbb{R}$ defined by $h_{\mu}^{(H)} \circ \pi_{\mu}^{(H)}=h \circ i_{\mu}^{(H)}$. The vector fields $X_{h}$ and $X_{h^{(H)}}$ are $\pi_{\mu}^{(H)}$-related. We will call $h_{\mu}^{(H)}$ the reduced Hamiltonian. Let $k: M \rightarrow \mathbb{R}$ be another $G$-invariant function. Then $\{h, k\}$ is also $G$-invariant and $\{h, k\}_{\mu}^{(H)}=\left\{h_{\mu}^{(H)}, k_{\mu}^{(H)}\right\}_{M_{\mu}^{(H)}}$, where $\{\cdot, \cdot\}_{M_{\mu^{*}}^{(H)}}$ denotes the Poisson bracket induced by the symplectic structure in $M_{\mu}^{(H)}$.

(iii) Let $\mathrm{N}(H):=\left\{n \in G \mid n H n^{-1}=H\right\}$ be the normalizer of $H$ in $G$. The Lie group $L:=\mathrm{N}(H) / H$, whose Lie algebra is denoted by $\mathfrak{l}$, acts freely and properly on $M_{H}$. Moreover, if $\mathrm{N}(H)$ is compact, this action is canonical and has an associated equivariant momentum map $\mathbf{K}_{L}$ given by the expression

$$
\mathbf{K}_{L}(z)=\left(\Lambda^{*} \circ \rho\right)\left(\mathbf{J}_{\left.\right|_{M H}}(z)\right)
$$

where $\Lambda^{*}$ is the natural $L$-equivariant isomorphism

$$
\Lambda^{*}:\left(\mathfrak{h}^{\circ}\right)^{H} \longrightarrow \mathfrak{l}^{*}
$$

between the $H$-fixed points $\left(\mathfrak{h}^{\circ}\right)^{H}$ of the annihilator $\mathfrak{h}^{\circ}$ of $\mathfrak{h}$ in $\mathfrak{g}^{*}$ and the dual $\mathfrak{l}^{*}$ of $\mathfrak{l}$, and

$$
\rho:\left(\mathfrak{g}^{*}\right)^{H} \longrightarrow\left(\mathfrak{h}^{\circ}\right)^{H}
$$

is the natural $L$-equivariant projection associated to an $\operatorname{Ad}(\mathrm{N}(H))$-invariant inner product in $\mathfrak{g}^{*}$ :

$$
\mathfrak{g}^{*}=\mathfrak{h}^{*} \perp \mathfrak{s}^{*}
$$

The subspace $\mathfrak{s}^{*}$ is the orthocomplement of $\mathfrak{h}^{*}$ in $\mathfrak{g}^{*}$ according to the inner product and the symbol $\perp$ denotes orthogonal direct sum. If $\mathbf{J}(m)=\mu$, we will write $\mu=\mu_{\mathfrak{h}^{*}} \perp \mu_{\mathfrak{S}^{*}}$ for the decomposition of $\mu$ according to the splitting (1), and we will call $\lambda_{\circ}=\Lambda^{*}\left(\mu_{\mathfrak{g}^{*}}\right)=\mathbf{K}_{L}(m)$. 


\section{J.-P. Ortega and T. S. Ratiu}

(iv) The reduced symplectic space $\left(M_{\mu}^{(H)}, \omega_{\mu}^{(H)}\right)$ is naturally symplectomorphic to the MarsdenWeinstein reduced space $\left(\mathbf{K}_{L}{ }^{-1}\left(\lambda_{0}\right) / L_{\lambda_{0}}, \omega_{\lambda_{0}}\right)$, corresponding to the $L$-action on $M_{H}$. The proof of this result consists basically in noting that $\mathbf{K}_{L}^{-1}\left(\lambda_{\circ}\right)=\mathbf{J}^{-1}(\mu) \cap M_{H}$ and that $L_{\lambda_{\circ}}=\mathrm{N}_{G_{\mu^{*}}}(H) / H$. Hence we have

$$
\mathbf{K}_{L}^{-1}\left(\lambda_{\circ}\right) / L_{\lambda_{0}}=\left(\mathbf{J}^{-1}(\mu) \cap M_{H}\right) /\left(\mathrm{N}_{G_{\mu}}(H) / H\right)
$$

The space on the right hand side of this expression, as we pointed out before, is diffeomorphic to $M_{\mu}^{(H)}$.

\section{Persistence}

The relative equilibrium with symmetry $m \in M$ is non-degenerate, if the corresponding reduced equilibrium $[m]_{\mu}^{(H)} \in M_{\mu}^{(H)}$ is such that $d^{2} h_{\mu}^{(H)}\left([m]_{\mu}^{(H)}\right)$ is a non-degenerate quadratic form. The Weyl group of $\mathrm{N}_{G_{\mu}}(H) / H$ will be denoted by $\mathrm{W}_{\mu}^{(H)}$, and the cardinality of the orbit $\mathrm{W}_{\mu}^{(H)} \cdot \eta$ through $\eta \in\left(\mathfrak{h}^{\circ}\right)^{H}$ by $\mathcal{W}_{\mu}^{(H)}(\eta)$.

THEOREM 1. - Let $(M, \omega, h)$ be a Hamiltonian $G$-space, with $G$ a Lie group acting properly on $M$, and $\mathbf{J}: M \rightarrow \mathfrak{g}^{*}$ the associated equivariant momentum map. Suppose that $m \in M$ is a nondegenerate relative equilibrium with $G_{m}=H$, such that $\mathrm{N}(H)$ is compact and $\mathbf{J}(m)=\mu$. Then, there is a $\mathrm{N}_{G_{\mu}}(H)$-invariant neighborhood $V \subset\left(\mathfrak{h}^{\circ}\right)^{H}$ of the origin such that for each $\alpha=\mu+\eta \in \mu+V$, there are at least $\frac{1}{2} \operatorname{dim}\left(\mathrm{N}_{G_{\mu}}(H) \cdot \eta\right)+1$ relative equilibria in $M_{\alpha x}^{(H)}$, or $\mathcal{W}_{\mu}^{(H)}(\eta)$ if they are all non-degenerate.

Proof. - By using the symplectomorphism introduced in point (iv), we will reduce the singular problem to the regular case described in [5].

The $G$-invariance of $h$, implies that the velocity of the relative equilibrium $m$ lies in $\operatorname{Lie}\left(\mathrm{N}_{G_{\mu}}(H)\right)$; moreover, there is a unique $\lambda \in \operatorname{Lie}\left(\mathrm{N}_{G_{\mu}}(H) / H\right)=\operatorname{Lie}\left(L_{\lambda_{0}}\right)$ such that $F_{t}(m)=\exp _{L} t \lambda \cdot m$, with $F_{t}$ the Hamiltonian flow associated to $h$ and $\exp _{L}: l \rightarrow L$ the exponential map. All this guarantees that $m$ is also a relative equilibrium of the Hamiltonian $L$-space $\left(M_{H}, \omega_{\left.\right|_{M_{H}}}, h_{\left.\right|_{M_{H}}}\right)$, which satisfies the hypothesis of Theorem 3.1 in [5] because the $L$-action on $M_{H}$ is free. Note also that $M_{\mu}^{(H)} \simeq \mathbf{K}_{L}^{-1}\left(\lambda_{0}\right) / L_{\lambda_{0}}=\left(M_{H}\right)_{\lambda_{0}}$ shows that $[m]_{\lambda_{0}}$ is non-degenerate in $\left(M_{H}\right)_{\lambda_{0}}$. Hence, we know that there is a $L_{\lambda_{0}}$-invariant neighborhood $U$ of 0 in $\operatorname{Lie}\left(L_{\lambda_{0}}\right)=\operatorname{Lie}\left(\mathbf{N}_{G_{\mu}}(H) / H\right)$ such that for each $\beta \in U$, there exist at least $\frac{1}{2} \operatorname{dim}\left(\mathrm{N}_{G_{\mu}}(H) / H \cdot \beta\right)+1$ relative equilibria in $\left(M_{H}\right)_{\lambda_{0}+\beta}$, or $\mathcal{W}_{\mu}^{(H)}(\beta)$ if they are all non-degenerate, with $\mathcal{W}_{\mu}^{(H)}(\beta)$ the cardinality of the orbit of $\beta \in \operatorname{Lie}\left(\mathrm{N}_{G_{H}}(H) / H\right)$ under the Weyl group of $\mathrm{N}_{G_{\mu}}(H) / H$.

Note that these are also relative equilibria for $(M, \omega, h)$ with $\mathbf{J}$ momentum equal to $\mu+\left(\Lambda^{*}\right)^{-1}(\beta)$. If we take $V$ such that $U=\Lambda^{*}(V)$ and $\eta$ satisfying $\beta=\Lambda^{*}(\eta)$, the result follows.

With the same setup used for relative equilibria, it is very easy to generalize the persistence result dealing with RPOs (see [4]), originally proved for free actions. We will say that a RPO is nondegenerate if the algebraic multiplicity of 1 as an eigenvalue of the Floquet operator corresponding to the associated periodic orbit in the singular reduced space is minimal, that is, 2 .

THEOREM 2. - Let $\gamma$ be a non-degenerate relative periodic orbit of the Hamiltonian system in Theorem 1 of energy $E_{0}$ and period $T_{0}$. Assume $m \in \gamma,[m]_{\mu}^{(H)} \in M_{\mu}^{(H)}$, and $\mathrm{N}(H)$ is connected. Then there is a $\mathrm{N}_{G_{\mu}}(H)$-invariant neighborhood $V \subset\left(\mathfrak{h}^{\circ}\right)^{H}$ of the origin such that for each $\alpha=\mu+\eta \in \mu+V$ and each $E$ close to $E_{0}$, there is at least one relative periodic orbit in $M_{(H)}^{\alpha}$ of energy $E$ and period close to $T_{0}$, or $\mathcal{W}_{\mu}^{(H)}(\eta)$ relative periodic orbits if they are all non-degenerate. 
Persistence and smoothness of critical relative elements in Hamiltonian systems with symmetry

Proof. - We use the symplectomorphism of point (iv) to describe $m$ as an RPO of the Hamiltonian $L$-space $\left(M_{H}, \omega_{\left.\right|_{1 \prime \prime},}, h_{\left.\right|_{M} \prime \prime}\right)$, which satisfies the hypothesis of Théorème 1 in [4]. Choices for $\eta$ and $V$ identical to the previous theorem complete the proof.

\section{Smoothness}

Patrick (see [8]) was the first to prove that for free actions with compact groups, the set of relative equilibria around a given one with regular velocity and momentum is a local symplectic manifold of dimension $\operatorname{rank}(G)$ in $M / G$. In the singular case, $M / G$ is not even a manifold in general. Thus, the natural setup is to study the relative equilibria lying in $M_{(H)} / G$. We will call them the set of relative equilibria of orbit type $(H)$. As we shall see below, under analogous strong hypotheses on the genericity of various Lie algebraic elements, the result of Theorem 1 can be vastly improved by obtaining a local symplectic submanifold consisting entirely of relative equilibria.

THEOREM 3. - Let $(M, \omega, h)$ be a Hamiltonian $G$-space with $G$ acting properly on $M$ and let $m \in M$ be a non-degenerate relative equilibrium with $\mathbf{J}(m)=\mu$ and $G_{m}=H$ such that $\mathrm{N}(H)$ is compact. If $\mu$ is such that $\lambda_{0}=\left(\Lambda^{*} \circ \rho\right)(\mu)$ is a regular element of $\mathfrak{l}^{*}$ and its velocity $\xi \in \operatorname{Lie}\left(\mathrm{N}_{G_{\mu}}(H)\right)$ can be chosen to be regular in the Lie algebra Lie $(\mathrm{N}(H))$ of $\mathrm{N}(H)$, then the relative equilibria of orbit type $(H)$ near $m$ form a smooth symplectic submanifold of dimension $\operatorname{dim} L+\operatorname{rank} L=\operatorname{dim} \mathrm{N}(H)+\operatorname{rank} \mathrm{N}(H)-2 \operatorname{dim} H$ in $M$.

Proof. - We will first need the following lemma whose proof is based on the Structure Theorem of compact Lie algebras.

LEMMA 1. - Let $\xi$ be a regular element in the Lie algebra $\mathfrak{n}=\operatorname{Lie}(\mathrm{N}(H))$. Then $\lambda=\mathrm{T}_{\epsilon} \pi(\xi) \in \mathfrak{l}=$ Lie $(\mathrm{N}(H) / H)$ is a regular element in $l$, where $\pi: \mathrm{N}(H) \rightarrow \mathrm{N}(H) / H$ is the canonical projection.

This lemma and the genericity condition on $\mu$ imply that $m$, understood as a relative equilibrium of the $L$-space $\left(M_{H}, \omega_{\left.\right|_{M_{H}}}, h_{\left.\right|_{M_{H}}}\right)$, satisfies Theorem 17 in [8] - recall that $\lambda_{\circ}=\left(\Lambda^{*} \circ \rho\right)(\mu)=\mathbf{K}_{L}(m)-$ which gives us a symplectic submanifold of relative equilibria of dimension $\operatorname{dim} L+\operatorname{rank} L$ that are also as relative equilibria of the system $(M, \omega, h)$.

Acknowledgements. We want to thank J. Montaldi for providing us with his papers well in advance of their publication and for many fruitful and illuminating discussions. Our thanks also to C. Dong, F. Fassò, E. Lerman, D. Lewis, J. Marsden, R. Montgomery, and M. Roberts for critically listening to us and giving us advice on various aspects of these problems.

Note remise le 23 juillet 1997, acceptée le 28 juillet 1997.

\section{References}

[1] Abraham R. et Marsden J. E., 1978. Foundations of Mechanics. Addison-Wesley, second edition.

[2] Bates L. et Lerman E. Proper group actions and symplectic stratified spaces. À paraître dans Pacific J. Math.

[3] Marsden J. E. et Weinstein A., 1974. Reduction of symplectic manifolds with symmetry. Rep. Math. Phys, 5, pp. 121-130.

[4] Montaldi J., 1997. Persistance d'orbites périodiques relatives dans les systèmes hamiltoniens symétriques. C. R. Acad. Sci. Paris, série I, 324, pp. 553-558.

[5] Montaldi J., 1997. Persistence and stability of relative equilibria. Nonlinearity, 10, pp. 449-466.

[6] Montaldi J. et Roberts M., 1997. Relative equilibria of molecules. Warwick Preprints, 6/1997.

[7] Ortega J.-P. et Ratiu T. S. Symmetry, Reduction, and Stability in Hamiltonian Systems, En préparation.

[8] Patrick G., 1995. Relative equilibria of Hamiltonian system with symmetry: linearization, smoothness, and drift. I. Nonlinear Sci., 5, pp. 373-418.

[9] Roberts R. M. et de Sousa Dias M. E. R. Bifurcations from relative equilibria of Hamiltonian systems. À paraître dans Nonlinearity.

[10] Sjamaar R. et Lerman E., 1991 Stratified symplectic spaces and reduction. Ann. Math. 134, pp. 375-422. 